\title{
The role of renewable energy in climate stabilization: results from the EMF27 scenarios
}

\author{
Gunnar Luderer ${ }^{1}$, Volker Krey ${ }^{2}$, Katherine Calvin ${ }^{3}$, James Merrick ${ }^{4}$, Silvana Mima ${ }^{5}$, Robert Pietzcker ${ }^{1}$, \\ Jasper Van Vliet ${ }^{6}$, Kenichi Wada ${ }^{7}$ \\ ${ }^{1}$ Potsdam Institute for Climate Impact Research, Potsdam, Germany \\ ${ }^{2}$ International Institute for Applied Systems Analysis, Laxenburg, Austria \\ 3 Joint Global Change Research Institute, College Park, MD, USA \\ ${ }^{4}$ Electric Power Research Institute, Palo Alto, CA, USA \\ ${ }^{5}$ Université Pierre-Mendès-France, Grenoble, France \\ ${ }^{6}$ PBL Netherlands Environmental Assessment Agency, Bilthoven, NL \\ ${ }^{7}$ Research Institute of Innovative Technology for the Earth, Kyoto, Japan
}

\begin{abstract}
This paper uses the EMF27 scenarios to explore the role of renewable energy in climate change mitigation. Renewables currently supply approximately $18 \%$ of global electricity demand. Almost all EMF27 mitigation scenarios show a strong increase in renewable power production, with a substantial ramp-up of wind and solar power deployment. In many scenarios, renewables are the most important long-term mitigation option for power supply. Wind energy is competitive even without climate policy, whereas the prospects of solar photovoltaics (PV) are highly contingent on the ambitiousness of climate policy. Bioenergy is an important and versatile energy carrier; however-with the exception of low temperature heat-there is less scope for renewables other than biomass for non-electric energy supply.
\end{abstract}

Despite the important role of wind and solar power in scenarios with full technology availability, limiting their deployment has a relatively small effect on climate mitigation costs. This is because they can be substituted by other low-carbon power supply options, such as nuclear or carbon capture and storage (CCS). Limited bioenergy availability in combination with limited wind and solar power, by contrast, results in a much more substantial increase in mitigation costs.

While a number of robust insights emerge, the results for renewable energy deployment levels vary significantly across the models. An in-depth analysis of a subset of EMF27 reveals substantial differences in modeling approaches and parameter assumptions. To a certain degree, differences in model results can be attributed to different assumptions about technology costs, resources, and systems integration. 


\section{Introduction}

There are multiple technological options for reducing greenhouse gas (GHG) emissions from the energy system. Besides renewable energy (RE) sources, carbon capture and storage (CCS) and nuclear energy are important supply-side mitigation options. RE is often praised as the most sustainable source of energy for two reasons. First, $\mathrm{RE}$ is, in principle, carbon-free. There are no direct $\mathrm{CO}_{2}$ emissions associated with the deployment of non-biomass RE, and the carbon emitted during the combustion of bioenergy is equal to the amount of carbon absorbed during plant growth. With a few exceptions, the life-cycle GHG emissions of RE sources are generally much lower than those caused by fossil fuels, even when all the stages of production are accounted for (Sathaye et al. 2011). Second, the defining feature of renewables is that their resource potential does not deplete over time. Moreover, the combined resource potential of all renewables exceeds the current energy demand by at least one order of magnitude (IPCC 2011). Given the constraints on fossil and nuclear fuel availability, geological reservoirs for $\mathrm{CO}_{2}$ storage, and the limited social acceptance of nuclear waste and onshore $\mathrm{CO}_{2}$ storage, it seems likely that the importance of RE will increase in the long-term even in the absence of climate policies. However, given the competition with other sources of energy, current RE deployment may be limited by relatively high costs, regional heterogeneity of resources (combined with limited transportability), and systems integration challenges.

The IPCC Special Report on Renewable Energy Sources and Climate Change Mitigation (SRREN) provides a comprehensive overview of the state of scientific knowledge on RE (IPCC 2011) by assessing, inter alia, resource potential, technology development, deployment costs, and potential future deployment levels. The assessment of the role of RE in model based climate mitigation scenarios performed for the SRREN (Krey and Clarke 2011) showed a strong expansion of RE in many scenarios as well as large differences across models.

The EMF27 study (Kriegler et al, this issue) provides a unique framework to further improve our understanding of the role of RE in climate change mitigation. It features a large set of scenarios with harmonized technology assumptions based on a wide ensemble of structurally different, state-of-the art integrated assessment models (IAMs). The goal of this paper is to analyze the role of RE in climate change mitigation using the EMF27 scenarios and to further qualify the determinants of RE-related differences in the model results. More specifically, we aim to answer the following research questions: (1) What RE deployment levels are consistent with various stabilization levels, and what are the roles of different RE technologies? (2) How can RE contribute to electric and non-electric energy supplies? (3) How does the availability of RE affect the cost and achievability of climate targets, and can ambitious climate targets be achieved through RE and energy efficiency alone? (4) What are the key model assumptions and uncertainties affecting RE deployment levels in mitigation scenarios?

The overview paper (Kriegler et al, this issue) provides a full description of the EMF27 scenario design. This paper focuses on the following technology variatons:

- AllTech: Default case with full technological availability

- LimSW: Share of electricity production from wind and solar limited to $20 \%$, and pessimistic assumptions regarding cost reductions of these energy sources. 
- LimBio: Primary energy supply from modern biomass limited to $100 \mathrm{EJ} / \mathrm{yr}$.

- Conv: Share of electricity production from wind and solar limited to $20 \%$, and primary energy supply from modern biomass limited to $100 \mathrm{EJ} / \mathrm{yr}$ (focus on conventional supply-side options).

- EERE: Unavailability of CCS, nuclear phase-out, and higher autonomous energy intensity improvement (30-45\% lower baseline final energy demand in 2100 compared to the other scenarios).

Each of these technology variations consider scenarios where atmospheric GHG concentrations are limited to $450 \mathrm{ppm} \mathrm{CO}_{2} \mathrm{e}$ by 2100 (temporary overshooting allowed), stabilize at $550 \mathrm{ppm} \mathrm{CO}_{2} \mathrm{e}$ (no overshoot allowed), or no climate policy is implemented (baseline).

\section{RE deployment across all models and specific scenarios}

The models differ significantly in their representations of RE. First, the models include different RE technologies. Table S2.1 in the supplementary material provides a detailed overview of the RE technologies represented in the models. While some models describe RE technologies with a high level of detail, e.g., by distinguishing between different solar and wind power technologies (TIAM-WORLD, MESSAGE, POLES, GCAM), other models with a stronger macro-economic focus only represent a few generic types of technology. In general, the models represent a wider variety of renewable options in the electricity sector than in the non-electric sector. Second, the models differ in terms of their methodological approaches and parameter assumptions. Differences related to renewable resource potentials, cost assumptions, and the consideration of systems integration are particularly relevant. It is important to keep these differences in mind when comparing scenario results. Section 4 examines the relationship between model assumptions and deployment levels for a subset of EMF27 models.

The remainder of this section reviews the renewable energy deployment levels in the EMF27 scenarios by assuming that all technologies are available and compares these deployment levels to the potentials provided in the literature. We find that total renewable energy deployment varies significantly across IAMs, both under baseline conditions and stringent mitigation policies.

\subsection{Hydropower}

Hydro electricity is currently the most significant non-biomass renewable energy source, supplying 12.7 EJ/yr or 16\% of the world's electricity in 2010 (IEA 2012). However, the technical potential for hydropower is limited to 50-60 EJ/yr (Kumar et al. 2011; Rogner et al. 2012; Turkenburg et al. 2012). As a result, growth in the deployment of hydropower is modest in most scenarios, with ReMIND, ENVLinkages, and AIM/CGE showing the most growth in the baseline (Base AllTech) scenario (Figure 1c). In most of the models, climate policy only results in a modest increase in deployment. TIAM-WORLD shows the largest response to climate policy, with deployment doubling in the 550 AllTech scenario. At the other extreme, many models show no change in the deployment of hydropower in response to climate policy. 


\subsection{Wind power}

The deployment of wind power in 2010 was considerably smaller than that of hydropower, accounting for $1.23 \mathrm{EJ} / \mathrm{yr}$ or $1.6 \%$ of global electricity generation (IEA, 2012). However, the resource potential of wind power is large and uncertain, with several studies citing 70-450 EJ/yr (Wiser et al. 2011; Rogner et al. 2012; Turkenburg et al. 2012) as the practical potential and as much as $5700 \mathrm{EJ} / \mathrm{yr}$ as the technical potential (GEA). ${ }^{1}$ The growth in deployment of wind power in the Base AllTech scenario is significant (Figure 1a), with most models showing an increase of 5-6\% per year throughout the century. As a result, deployment is substantial in many of the models (even in the baseline scenario), reaching $81 \mathrm{EJ} / \mathrm{yr}$ in the POLES model by the end of the century (see Figure SM2.1 in the supplementary material). Climate policy has a varied effect on wind power across all of the models - some models (e.g., EC-IAM, IMAGE) show lower deployment of wind power in absolute terms under a climate policy, while most models (e.g., AIM-Enduse, AIM/CGE, BET) show significant increases.

\subsection{Solar Power}

Although deployment of solar power has shown annual growth rates of almost $40 \%$ over the last ten years, the current deployment rate is still very small, supplying only $0.12 \mathrm{EJ} / \mathrm{yr}$ globally in 2010 (IEA). By contrast, the technical potential for solar power is enormous. Turkenburg et al. (2012) estimate the technical potential for photovoltaics (PV) to range from 1,600-50,000 EJ/yr. Similarly, Arvizu et al. (2011) estimate a technical potential of 1,338-14,778 EJ/yr for PV and a technical potential of 248-10,791 EJ/Y for concentrating solar power (CSP). Growth in the deployment of solar power in the Base AllTech scenario varies significantly across the models, ranging from 0-17 EJ/yr in 2050 and 0.3-143 EJ/yr in 2100. Climate policy increases solar power generation in most of the models, often substantially.

\subsection{Geothermal}

Conventional electricity generation from hydrothermal reservoirs is already mature, but at present, it plays a minor role in current energy systems, accounting for approximately $0.2 \%$ of global electricity generation in 2010 (IEA 2012). The resource potentials for hydrothermal electricity generation are limited: Goldstein et al. (2011) report a range of 28-56 EJ/yr. Enhanced geothermal systems (EGS) have a considerably larger resource potential (89-1052 EJ/yr as reported by Goldstein et al. (2011), but the technology is less mature. Many of the EMF27 models do not consider geothermal power, and others only represent it in a rather stylized way (e.g., REMIND). TIAM, BETS, AIM/CGE, and GCAM are the most optimistic about geothermal power, but feature deployment levels well below $10 \mathrm{EJ} / \mathrm{yr}$ (Figure 1d).

\subsection{Bioenergy}

As of today, bioenergy is the renewable energy source with the largest deployment level. The global consumption of bioenergy, including traditional biomass, was $53 \mathrm{EJ} / \mathrm{yr}$ in 2010, which accounts for more than $10 \%$ of total primary energy (IEA). Current bioenergy use is, however, dominated by traditional fuel

\footnotetext{
${ }^{1}$ Wiser et al. (2011) does not use the "practical" and "technical" distinction. Instead, the authors compare potential with "limited constraints" and "more constraints". They estimate 70-450 EJ/yr with more constraints and 70-3050 EJ/yr with limited constraints.
} 
use with low final to useful efficiency. The technical potential for bioenergy in 2050, as estimated by SRREN, is 50-1000 EJ/yr (Chum et al. 2011). Bioenergy is unique for two reasons: (1) its versatility (it can be used to produce liquids, electricity, hydrogen, gases, or heat) and (2) the possibility to create negative emissions when combined with CCS. The deployment of bioenergy technologies varies significantly across the models and scenarios in the EMF27 study (Figure 1e and f). For example, bioelectricity generation in the Base AllTech scenario varies from $0.03 \mathrm{EJ} / \mathrm{yr}$ (IMACLIM) to $10.5 \mathrm{EJ} / \mathrm{yr}$ (IMAGE) in 2050. The range is larger in $2100(0.001-27 \mathrm{EJ} / \mathrm{yr}$ ) and under a climate policy (0.3-33 EJ/yr in 2050 in the 450 AllTech scenario). The production of bioliquids in the Base AllTech scenario varies from 0 $\mathrm{EJ} / \mathrm{yr}$ (BET) to $27 \mathrm{EJ} / \mathrm{yr}$ (MERGE) in 2050. Again, this range increases in 2100 (0.4-89 EJ/yr) or if a climate policy is imposed (0-77 EJ/yr in 2050 in the 450 AllTech scenario). A limited number of models consider heat or gas produced from bioenergy. However, deployment of these technologies can be substantial with TIAM-World reaching $18 \mathrm{EJ} / \mathrm{yr}$ of heat from bioenergy and $35 \mathrm{EJ} / \mathrm{yr}$ of gas from bioenergy in 2050 under a climate policy. Rose et al. (this issue) discuss the deployment of bioenergy in the EMF27 scenarios.

\subsection{Other RE sources for heat supply}

Additional RE sources, aside from biomass, exist for the provision of non-electric energy, such as geothermal heat and solar heat. In 2010, the installed capacity for solar heat production was an order of magnitude larger than the capacity for solar electricity production (Arvizu et al. 2011). Deployment is substantial in climate policy cases in the few models that represent these options. For 450 AllTech, solar heating systems account for 16-34 EJ/yr in 2050 in POLES, AIM-CGE and MESSAGE, a substantial share of total heat supply. 

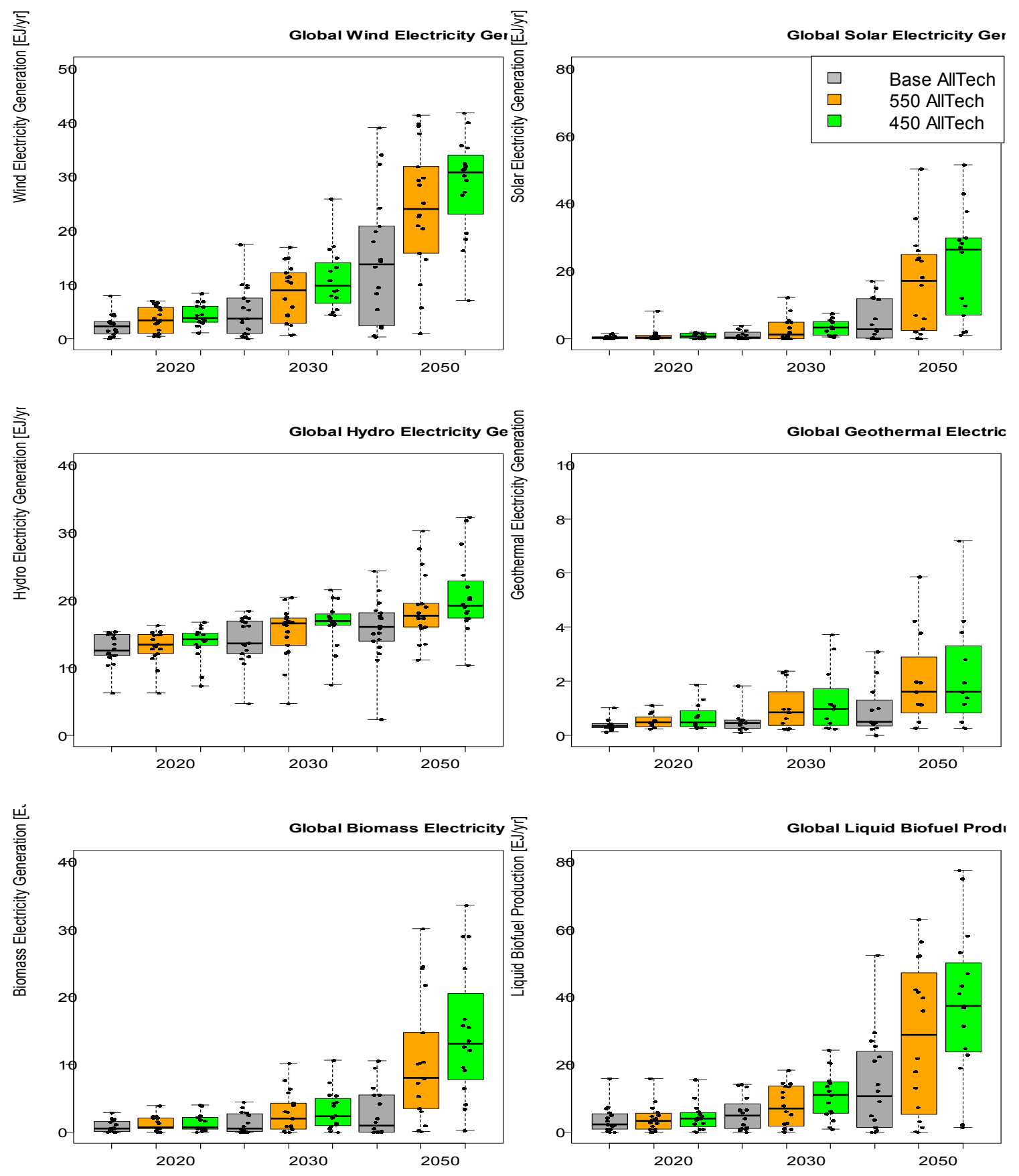

Figure 1. Deployment levels of various RE technologies in the full technology cases in the AllTech baseline 550 and 450 scenarios. 


\section{The relevance of $\mathbf{R E}$ for mitigation}

This section considers the energy system from a broader perspective in order to examine the relevance of RE for mitigation. The EMF27 scenarios allow us to study how RE deployment levels change with alternative technology assumptions, and how they substitute with alternative energy supply technologies and climate mitigation options. We focus on the $550 \mathrm{ppm}$ climate target because more models report results for technology-constrained scenarios for this stabilization level. By exploring RE deployment for electric and non-electric energy, we analyze in which areas what types of RE sources contribute most.

\subsection{The role of $R E$ in energy supplies}

Figure 2a shows electricity supplies in the 550 AllTech climate mitigation scenarios averaged between the years 2010-2100. The results indicate that renewables can play an important or even dominant role in electricity generation if climate policies are in place. While the RE contribution varies in the different models, all but one model have more than a 35\% share of RE in electricity in the second half of the century, and half of the models have an RE share of $59 \%$ or higher. The models with high overall RE deployment in the power supply, such as REMIND, MESSAGE, TIAM-WORLD, POLES and GRAPE, tend to have particularly large shares of solar and wind power, while the contribution of hydropower is more comparable across models. This is not surprising since the limitations on resource potential are less constraining for solar and wind power than for hydropower. In scenarios with high RE deployment, the share of fluctuating RE power supply from wind and solar PV exceeds $40 \%$ in the second half of the century. Systems-integration challenges associated with high shares of RE are discussed in Section 4.3. In the scenarios where bioenergy is used for electricity generation, it is mostly deployed with CCS in order to produce net negative emissions. Other models feature limited bioenergy use in the electricity sector. This is often driven by the high value of bioenergy for biofuel production.

Figure $3 \mathrm{~b}$ shows the conversion pathways for non-electric secondary energy sources. In contrast to electricity, non-electric energy remains dominated by fossil fuels even if climate policies are in place. Biomass is the most important supply-side mitigation option for non-electric energy. It is primarily used to produce liquid biofuel as a substitute for oil. In models that consider liquid biofuel production with and without CCS (GCAM, MESSAGE, REMIND, TIAM-WORLD), production processes with CCS dominate over conversion pathways without CCS in the long-term. Rose et al. (this issue) provide a detailed analysis of bioenergy use in the EMF scenarios. Solar-thermal and geo-thermal heating systems are potentially the most relevant non-biomass renewable options for non-electric energy sources. As discussed in Section 2.2.6, only a few EMF27 models consider these options. While deployment can be substantial for individual technologies, non-biomass renewables represent a very small share of nonelectric energy sources across all EMF27 scenarios. As a consequence, non-electric energy use declines more rapidly in scenarios with a climate policy as some of the current non-electric energy demand becomes electrified. 
(a) Electricity (2010-2100) - 550 AllTech

(b) Technology Shares in Electricity
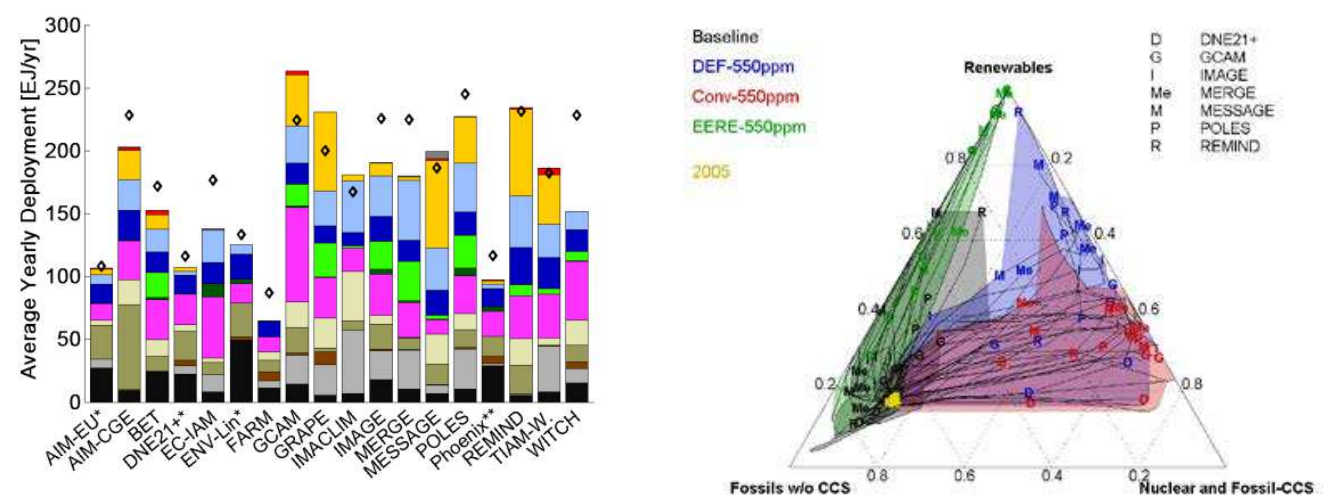

(c) Electricity (2010-2100) - 550 Conv

(d) Electricity (2010-2100) - 550 EERE
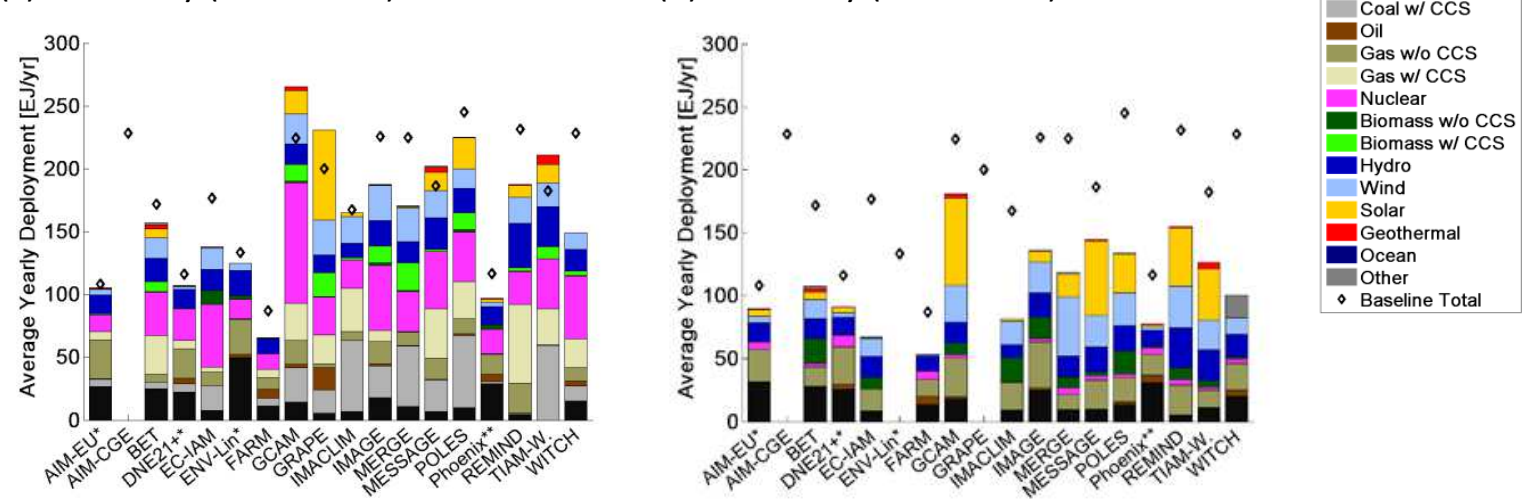

(e) Difference 550 Conv - 550 AllTech

(f) Difference 550 EERE - 550 AllTech
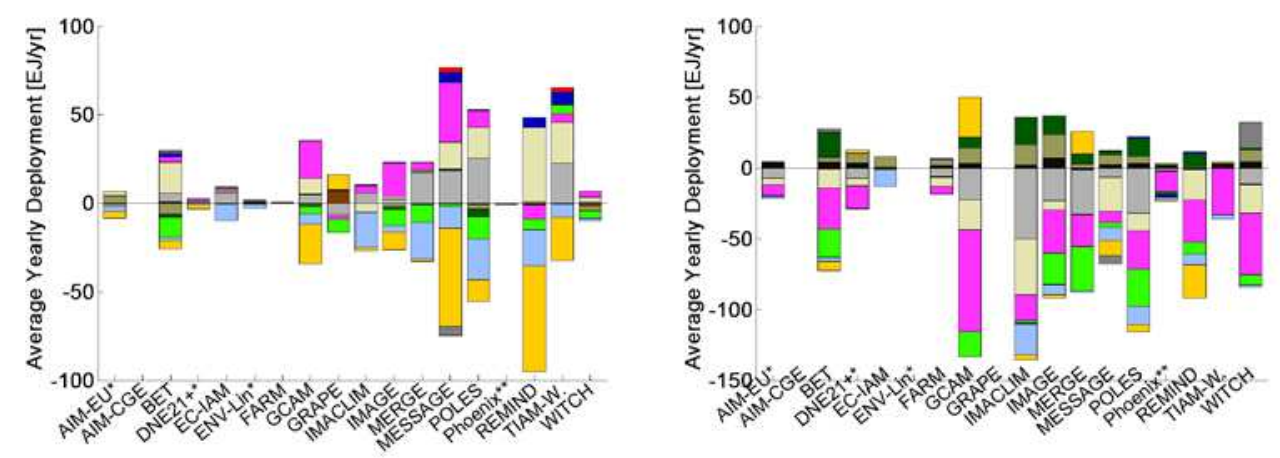

Figure 2. Average yearly electricity production from 2010-2100 for the 550 ppm climate stabilization scenarios with different technology assumptions: (a) AllTech, (c) Conv, and (d) EERE. Deployment differences between the (e) Conv and (f) EERE scenarios are relative to AllTech. The (b) ternary graph shows trajectories of technology shares over time for all EMF27 models, with a subset of models highlighted. *For AIM-EU, DNE21+, and ENV-Lin, a time span of 2010-2050 was considered. The diamond markers indicate totals in the Base AllTech scenarios. **For Phoenix, a time span of 2010-2070 was considered. 
(a) Non-electric (2010-2100) - 550 AllTech

(b) Technology Shares in Electricity
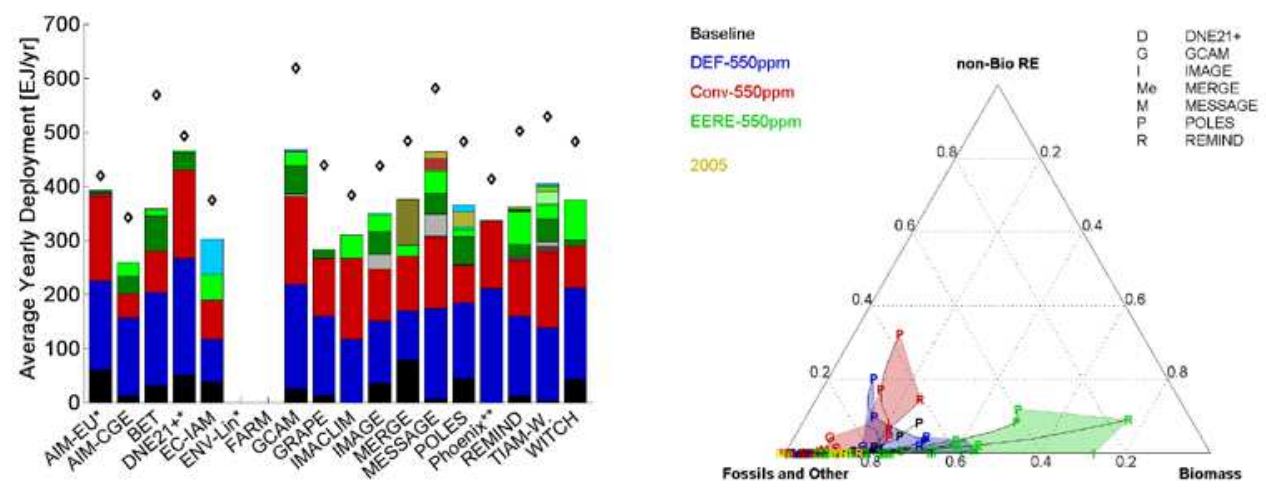

(c) Non-electric (2010-2100) - 550 Conv

(d) non-electric (2010-2100) - 550 EERE
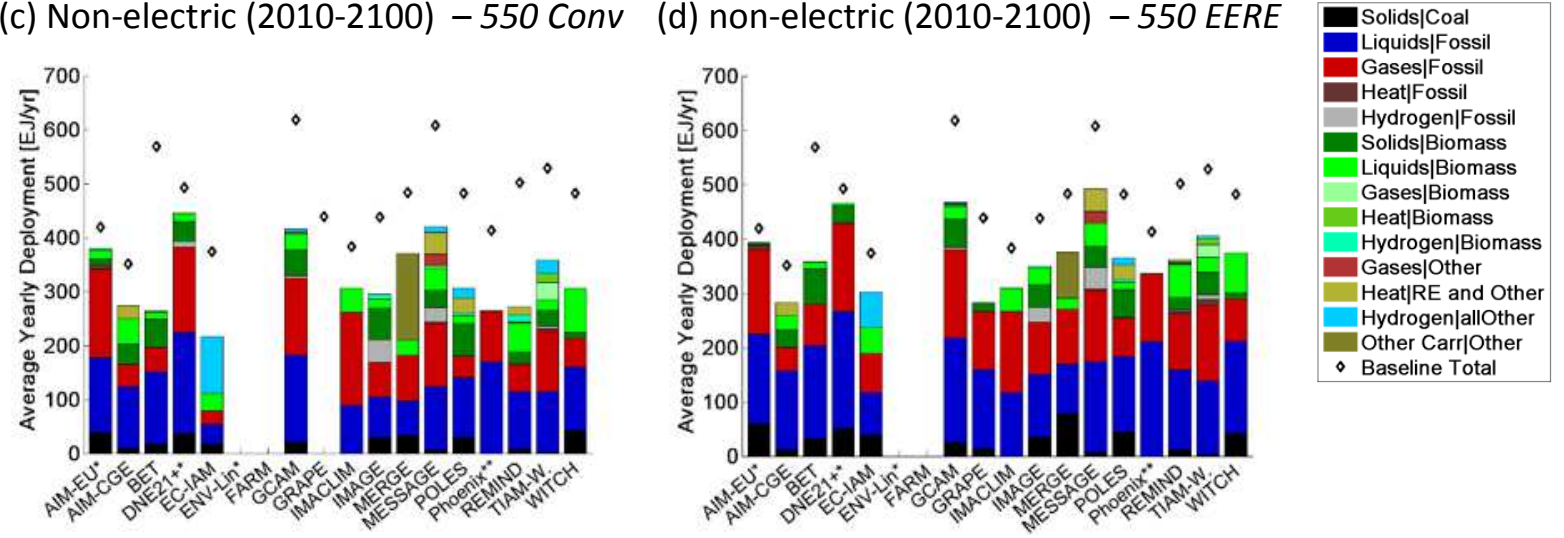

(e) Difference 550 Conv - 550 AllTech

(f) Difference 550 EERE - 550 AllTech

non-electric - 550 ppm--World (2005-2100)

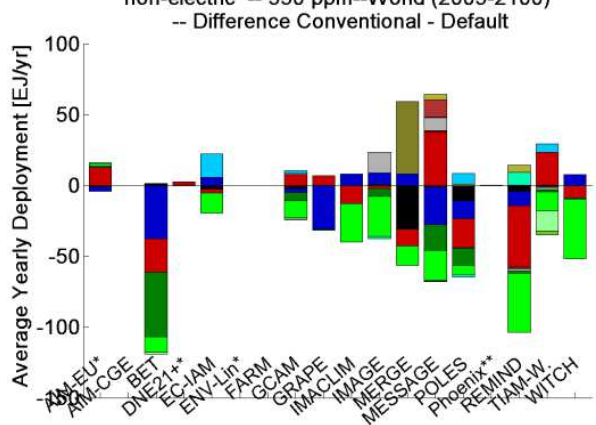
non-electric - 550 ppm--World (2005-2100)

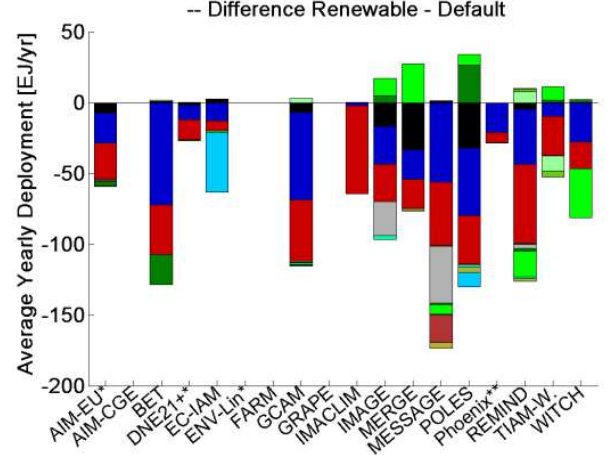

Figure 3. Non-electric secondary energy supply cumulated from 2010-2100 for the 550 ppm climate stabilization scenarios with different technology assumptions: (a) AllTech, (c) Conv, and (d) EERE. Difference deployment between the (e) Conv and (f) EERE scenarios are relative to AllTech. The (b) ternary graph shows the trajectories of technology group shares over time for all EMF27 models with a subset of models highlighted. *For AIM-EU, DNE21+, and ENV-Lin, a time span of 2010-2050 was considered. ${ }^{* *}$ For Phoenix, a time span of 2010-2070 was considered. 


\subsection{Substitution between RE and other low-carbon supply options}

In addition to the default scenarios with full technology availability, there are two technology variations that are of particular interest for elucidating the role of RE. These scenarios allow us to determine if the large-scale deployment of wind, solar, and bioenergy power is critical for climate change mitigation. Using the Energy Efficiency and Renewable (EERE) scenarios, we can determine if mitigation targets can be reached solely using energy efficiency and renewables.

In the AllTech climate policy scenarios, the models agree on the strong decrease of fossil-based electricity without CCS, but show a variety of decarbonization pathways (Figure $2 \mathrm{a}$ and $\mathrm{b}$ ). For some models (REMIND, MESSAGE, POLES, TIAM-WORLD), nuclear and CCS are mostly relevant in the mediumterm, while power supply is dominated by RE in the long-term. In other models (WITCH, AIM/CGE, ECIAM, IMACLIM), nuclear, RE, and CCS contribute in roughly equal shares throughout the century. Electricity supply is very responsive to the technology variations in the EMF27 scenarios. CCS, nuclear, and renewables are alternative low-carbon options that represent good substitutes in carbonconstrained scenarios. The limitations on wind, solar, and bioenergy use imposed in the Conv scenario result in higher deployment of CCS and nuclear (Figure 2e). Similarly, more wind, solar, and CCS technologies are used in the nuclear phase-out scenarios (NucOff; cf. Krey et al., this issue) while more wind, solar, and nuclear use results from the unavailability of CCS (NoCCS; cf. Krey et al., this issue; Figure S3.1).

Limited bioenergy availability has a considerable impact on non-electric energy supply in the Conv scenario (Figure $3 c$ and e). In most models, the supply of liquids, gases and solids decreases substantially compared to the AllTech scenario. There are two main reasons for this pattern. First, there is a lack of non-electric low-carbon substitutes for biofuels in most models. ${ }^{2}$ Second, bioenergy has the potential to create negative emissions by combining it with CCS (BECCS; see also Rose et al., this issue). Reducing bioenergy availability results in less negative emissions, resulting in less leeway for the continued use of fossil fuels for non-electric energy.

In the EERE scenario, renewables are the only long-term low-carbon options for electricity supply. On the other hand, the lower energy demand due to the assumption of lower energy largely compensates for the lack of electricity from nuclear and CCS. This results in relatively small deployment differences relative to the AllTech scenario for renewable technologies. However, in many models, more coal and gas without CCS are used for electricity supply, resulting in a higher share of freely emitting sources than in AllTech. At the same time, the lack of the BECCS option to create negative emissions decreases the cumulative fossil use that is permissible within the climate constraint. This restriction results in additional reductions in fossil fuel use for non-electric energy in the 550 EERE scenario. Biofuels increase only slightly in absolute terms, but their share is substantially higher than in the AllTech scenario.

\footnotetext{
${ }^{2}$ MERGE and EC-IAM are an important exception as they represent generic carbon-free backstop technologies for non-electric energy or hydrogen.
} 


\subsection{The impact of RE availability on mitigation costs}

The different roles that low-carbon energy technologies play in electric and non-electric energy are important factors for explaining the impact of technology constraints on the costs and feasibility of climate targets. Figure 4 displays the costs of reaching the 550 and 450 ppm climate policy targets under limited technology scenarios normalized to the costs in the corresponding AllTech scenarios. For the Conv scenarios, the EMF27 models show that limited availability of wind, solar, and bioenergy results in a substantial cost increase. This finding is in line with earlier studies, which found similar cost increases by examining climate policy scenarios with restrictions on the expansion of RE (Edenhofer et al. 2010; Pugh et al. 2011; Luderer et al. 2012). The EMF scenarios allow us to separate the effects of bioenergy availability (LimBio scenario) from limitations on wind and solar-power use (LimSW scenario). The models consistently find higher cost penalties for limiting biomass than for limiting solar and wind power in both the 550 and $450 \mathrm{ppm}$ climate mitigation scenarios. This is explained by the fact that in case of limitations on wind and solar power other low-carbon alternatives such as nuclear or CCS are readily available, while biomass has a more unique role in the climate mitigation effort (see discussion in Sections 2.5 and 3.2). The cost increases escalate if biomass, and wind and solar use are limited.

(a) 550 ppm scenarios

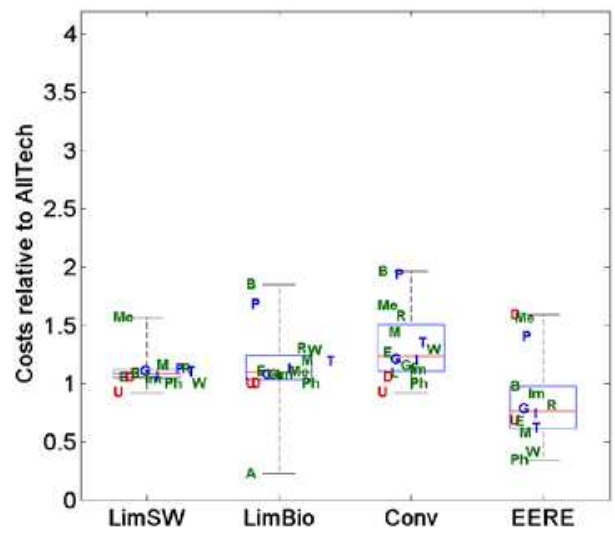

(b) 450 ppm Scenarios

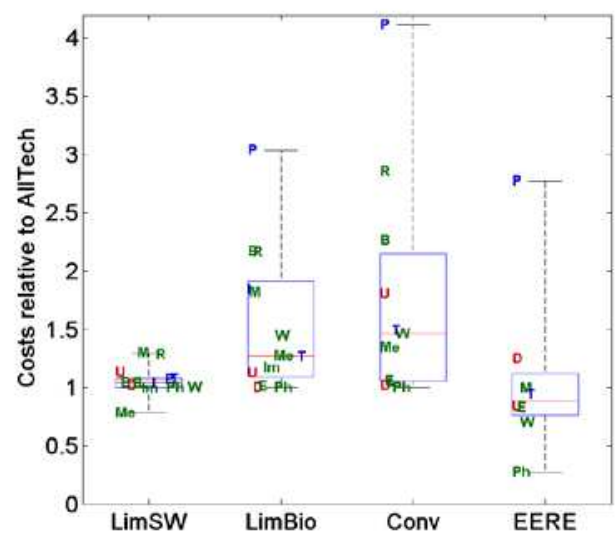

U AIM-EU

A AIM-CCEE
B BET

DNE21

EC-IAM

ENV-Li

GCAM

II IMACLIM

IMAGE
Me MERGE

$M$ MESSAGE

Ph Phoenix

$P$ POLES

R REMIND

TIAM

W WITCH

Area under MAC Curve

Consumption Loss Energy System Cost

Figure 4. Climate policy costs for scenarios with reduced technology portfolios, indexed relative to the corresponding AllTech scenario.

The EERE scenarios, which rely solely on energy efficiency and renewables for mitigation, offer a complementary perspective on the role of renewables for climate change mitigation. In terms of policy costs, two forces are at play. On one hand, the lower baseline energy demand results in lower baseline emissions, and thus, a smaller mitigation gap towards the climate target. On the other hand, the unavailability of CCS and nuclear makes the mitigation effort more difficult than in the AllTech scenarios. This explains the wide range of policy cost outcomes. In the 550-ppm case, all the models except DNE21, MERGE, and POLES show lower costs in the EERE scenario than in AllTech. In the 450-ppm scenario, the split becomes more extreme: almost half the models found the $450 \mathrm{ppm}$ target infeasible in the $E E R E$ 
setting, while in most of the other models (such as WITCH and Phoenix), policy costs in the EERE scenario are lower than in AllTech.

\section{Determinants of wind and solar power deployment}

As noted in Section 2, the observed deployment levels of different renewable energy sources differ strongly across the models participating in EMF27. The objective of this section is to relate RE deployment levels to model assumptions and characteristics. This section discusses the key determinants of deployment levels, including resource availability, various types of technology costs (e.g., investment, operation, and maintenance costs, or levelized costs of electricity generation [LCOE] as an aggregate cost measure), and systems-integration constraints. Detailed information and data about RE parameters and assumptions are available for the seven models that participated in the EMF27 RE subgroup (DNE21+, GCAM, IMAGE, MERGE, MESSAGE, POLES and REMIND). The diagnostic analysis in this section focuses on these models as well as China and the USA.

\subsection{Technology costs and competition with other technologies}

Technology choices in energy-economic models are typically the result of a cost minimizing or welfare maximizing optimization procedure, or an explicit selection based on levelized costs. It can, therefore, be expected that the role of RE for climate change mitigation primarily depends on its competitiveness vis-à-vis nuclear and CCS, which represent the other two important low-carbon options for electricity supply.

Since no fuel costs are incurred for wind and solar power, deployment costs are largely driven by capital cost. Most models project capital costs in the range of 1000-1400 $\$ / \mathrm{kW}$ for solar PV and 750-1000 $\$ / \mathrm{kW}$ for wind onshore in 2050 (Table S2.1 supplementary material). For IMAGE, capital costs for onshore wind turbines are considerably lower than in the other models. REMIND, IMAGE, and POLES treat technological learning endogenously, resulting in lower capital costs in the policy scenarios compared to the baseline.

Figure 5 contrasts LCOE and deployment levels for solar, wind, nuclear, gas CCS, and coal CCS in the USA and China in 2050 for the 450-ppm AllTech scenario. Deployment levels roughly mirror LCOE patterns in the sense that technologies with lower LCOEs are tend to be deployed at higher levels. In all the models, wind power is competitive with or even cheaper than electricity production from nuclear or fossil CCS plants. However, it must be noted that the LCOEs shown in Figure 5 do not reflect the costs related to the integration of electricity from wind and solar with variable and non-dispatchable output. Moreover, it is important to note that, in addition to the influence of the costs of renewable technologies, the deployment of these technologies can be affected by the modeling of capital stocks through implicit or explicit constraints on the amount of capital available. For example, an expansion constraint in the MERGE model limits the rate at which new technologies can be up-scaled.

For all the models except DNE21+, wind power deployment is considerable in the USA and China, and solar energy tends to be more expensive than wind power. In some of the models, resource limitations 
are a constraint for wind deployment in China, which explains the low deployment levels in DNE21+ (see Section 4.2).

Due to high costs, solar power is not deployed in MERGE and remains insignificant in IMAGE. Solar power is more important in the other models, with more than $5 \mathrm{EJ} / \mathrm{yr}$ of solar supply in China. In REMIND, which operates under perfect foresight, the anticipation of benefits from technological learning results in an earlier and higher deployment of solar PV, despite temporarily higher LCOEs.

(a) USA - 450 AllTech - 2050
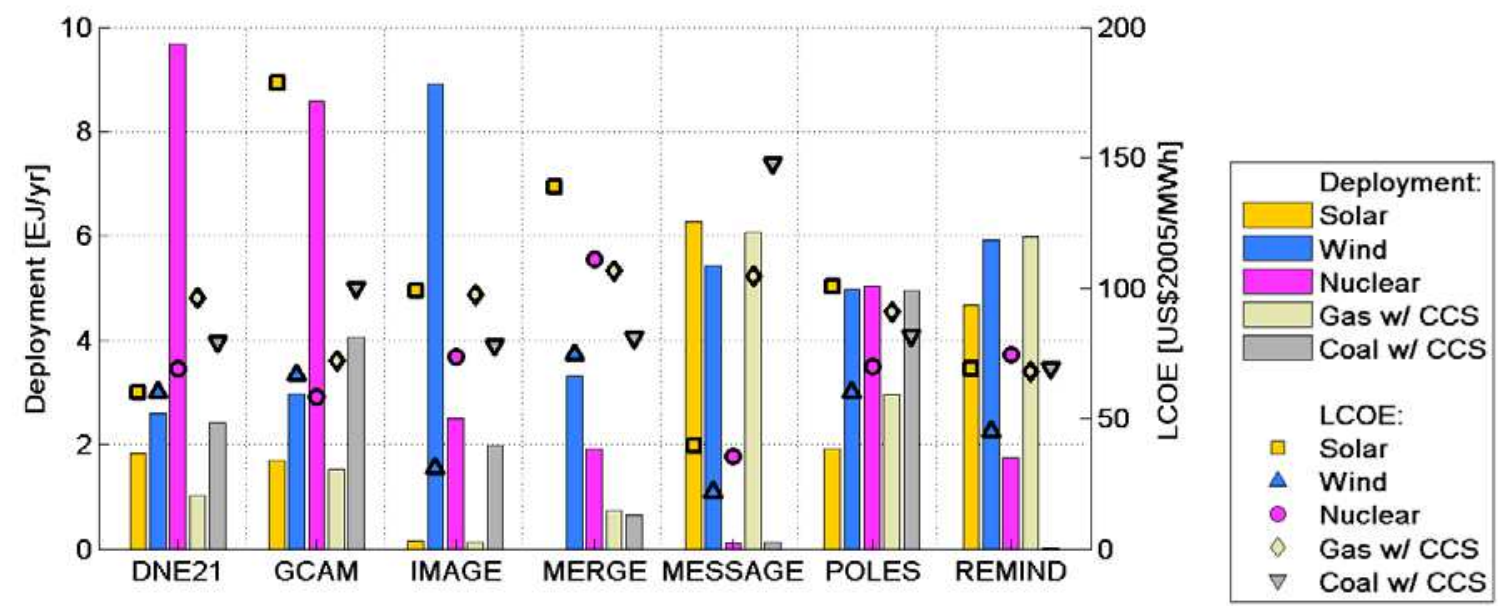

(b) China -450 AllTech -2050

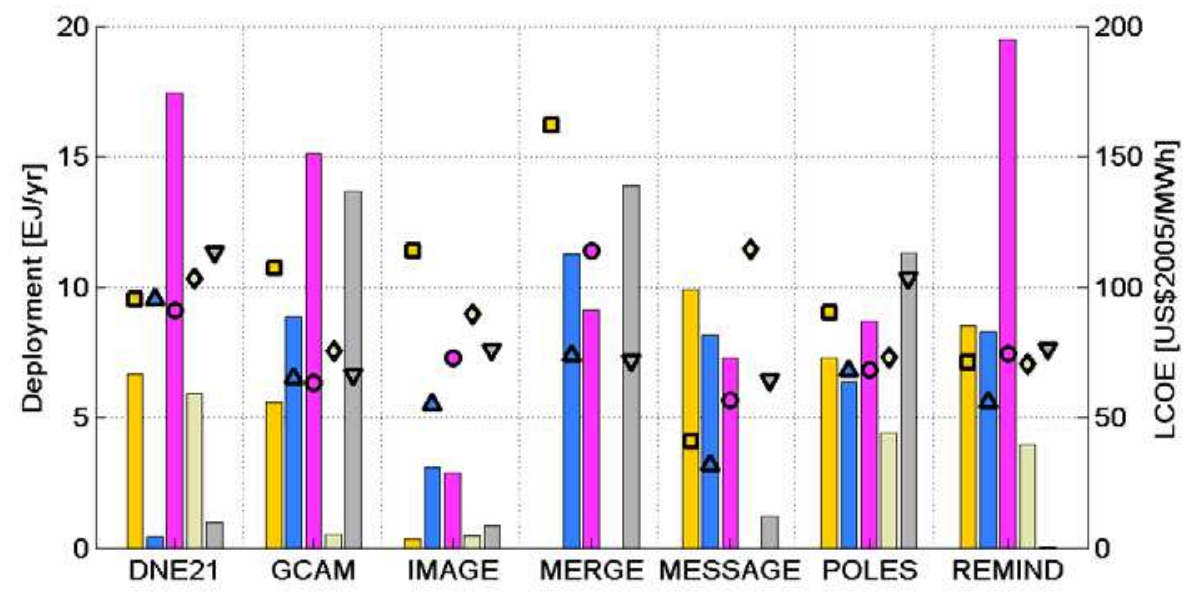

Figure 5. Deployment levels of selected low-carbon technologies (bars, left axis) and corresponding average LCOE (markers, right axis) for the 450-AllTech Scenario in 2050. Upper row: USA; lower row: China. 


\subsection{Renewable energy resource potentials}

Another key determinant for RE deployment is the resource availability. Figure 6 shows the onshore wind and solar PV resource potentials for the selected models in the USA and China next to the respective deployment pathways in the 450 AllTech scenario. This comparison reveals that resource potentials at the regional level vastly differ across models. In both regions, the lowest and highest resource potential estimates differ by more than one order of magnitude across the models and in some cases, turn out to be binding for the observed deployment levels. In addition, resource qualitycharacterized by the capacity factors (see blue shadings in Figure 6 and Figures S4.1-S4.3)-varies across the data sets by a factor of two in the best resource categories represented in the models. When comparing deployment levels with resource potentials at the regional level, the renewable resource data adopted by the different IAMs can explain some of the differences in the deployment of onshore wind turbines. For example, the upper end of the regional supply curve determines the maximum deployment in at least one model (MERGE), but others are close to the maximum deployment level as well. In addition, data resolution in the supply curves in some cases are a governing factor for the deployment pattern of the resource. ${ }^{3} \mathrm{~A}$ similar comparison for solar PV shows that the resource potentials included in the models tend to be significantly higher than for wind (with the exception of POLES, where only rooftop PV is considered). Therefore, resource availability is not a limiting factor for technology deployment. Instead, cost assumptions as well as competition with other technologies are much more relevant (Section 4.1).

In part, the differences in resource potentials are due to definitional issues, which make them difficult to compare. However, it is possible to trace many of these differences back to the original resource data sets employed by the models, which in turn, are based on different methodologies and show a considerable spread. The supplementary material (SM4.2) further discusses the underlying resource data sets.

\footnotetext{
${ }^{3}$ Note that the native supply curve steps are not necessarily identical to the ones shown in Figure 6 for which an aggregation to 5\%-point-steps is attempted to allow for an easier comparison.
} 

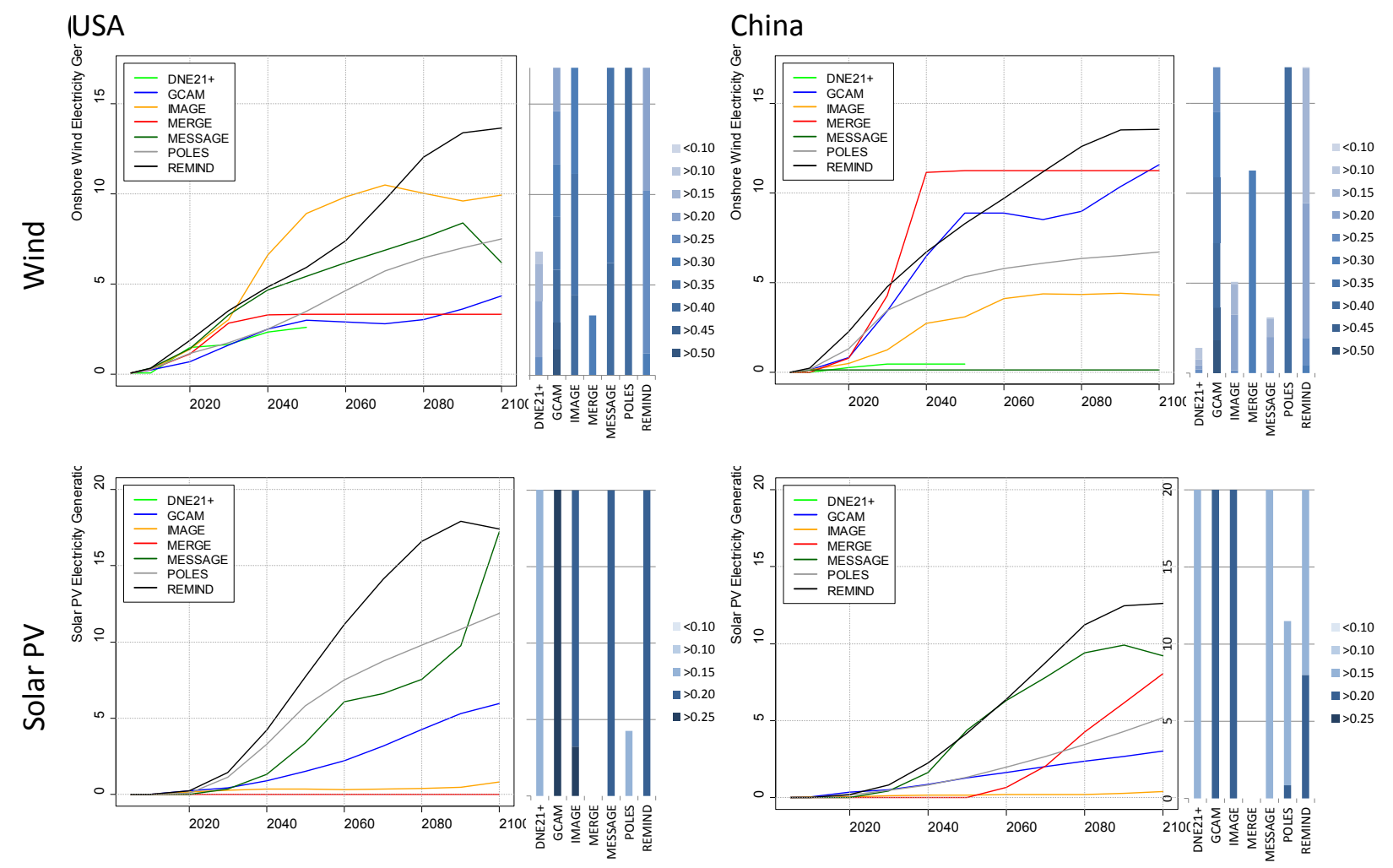

Figure 6. Wind deployment and resource potentials by capacity factor in (a) the USA and (b) China in the 450 AllTech scenario from selected models participating in the EMF27 study. Note that regional definitions are not comparable in all cases and that for some models, offshore wind data are combined with onshore wind (see supplementary material).

\subsection{Systems Integration}

One crucial drawback of wind and solar power is the spatial and temporal variability of their outputs. Many of the EMF27 scenarios describe electricity systems with high a penetration of renewable intermittent generation in excess of $20 \%$. The need to match load and supply at all times in such systems requires major changes to the operation and design of current electricity systems.

RE fluctuations operate on time scales that are much smaller than those resolved by IAMs, which typically focus on long-term transformations measured in years. Therefore, these models represent RE integration challenges in a rather stylized way. Table S4.3 provides an overview of the systemsintegration mechanisms represented in the models. The most basic approach to reflecting integration challenges is to set a constraint on the maximum share of wind and solar power in electricity generation. For instance, BET limits the combined share of wind and solar to $30 \%$. Similar constraints are implemented in AIM-Enduse, BET, EC-IAM, FARM, GRAPE, and IMACLIM. These models tend have relatively low overall RE shares in the electricity supply. A disadvantage of hard constraints is that they are price-insensitive and neglect the possibility for system adjustments under strong economic pressure. 
Other approaches make the economic trade-offs related to RE integration more explicit by introducing cost penalties that increase with RE penetration by demonstrating how storage and backup requirements depend on RE penetration or by representing load duration curves. Many models use a combination of several approaches. The system-integration costs can be substantial. For instance, they amount to $23 \$ / \mathrm{MWh}$ at $20 \% \mathrm{PV}$ and $15 \%$ wind penetration for REMIND in the 450 AllTech scenario in the USA in 2050. Similarly, a cost penalty on wind deployment amounting to $15 \$ / M W h$ is applied in MERGE. In several of the models that explicitly consider integration challenges (POLES, TIAM, MESSAGE, and REMIND), wind and solar power combined account for more than $40 \%$ of electricity supply in the latter half of the $21^{\text {st }}$ century, despite high integration costs.

\section{Conclusions}

This paper analyzes the role of RE in climate change mitigation based on a large set of state-of-the-art IAMs and the coordinated scenario provided by the EMF27 study.

One important conclusion is that the relevance of RE sources is very different in the various energy supply sectors. Renewables can play an important or even dominant role in the power sector. In most models, the use of RE for electricity increases even without climate policies. In mitigation scenarios, RE deployment for electricity supply expands considerably, with an increasing share of wind power in all models and substantial long-term deployment of solar power in most models.

Another important insight from the EMF-study is that the decarbonization of fuels for transport, buildings and industry are crucial bottlenecks for reducing energy related emissions. Bioenergy is a versatile substitute for fossil fuels that can produce various energy carriers, and therefore is by far the most important mitigation option for non-electric energy production. The EMF27 scenarios suggest that renewable power in combination with electrification of end-use (e.g. via electric vehicles, electric arc furnaces, or geothermal heat pumps) is an important mitigation option. Beyond electrification, renewables can contribute via low-temperature heat. Solar-thermal energy systems account for a substantial share of heat supply in the few models in which they are represented. Given the potential importance of renewable heat supply, a broader and more refined representation in IAMs as well as efforts to improve bottom-up estimates of their deployment potential seem desirable.

Restricting the penetration of wind and solar energy to $20 \%$ of electricity supply has a relatively small effect on the costs of climate policy. This is not a surprise, given the ample availability of other technologies for low-carbon electricity, such as nuclear and CCS. In contrast, limiting the availability of bioenergy to $100 \mathrm{EJ} / \mathrm{yr}$ results in significantly higher cost increases not only because of its importance for decarbonizing non-electric energy supply, but also the possibility of generating negative emissions by combining bioenergy production with CCS. The EMF27 scenarios also show that it is very difficult to reach the $450 \mathrm{ppm}$ climate target by relying on energy efficiency and renewable energy alone, i.e., without CCS and nuclear energy.

While many of the findings regarding the potential role of RE for climate mitigation are rather robust, the deployment levels of individual technologies vary considerably across models. An in-depth analysis based on a subset of EMF27 models shows that the diversity of the results mirrors the wide range of 
assumptions on crucial parameters. In particular, there is a substantial discrepancy between the RE resource assumptions used in the models. Therefore, it is necessary to derive new global resource data sets for the most frequently discussed options (e.g., wind, solar PV, CSP) as well as for the less wellrepresented options (e.g., solar heat, geothermal heat). Moreover, there is substantial uncertainty about the future evolution of technology costs for RE and relevant competing low-carbon technologies. In the past, renewable technologies have shown considerable cost reduction potential. Improved estimates of future costs and making related uncertainties explicit will be important to further improve our understanding of the role of RE. Finally, spatial and temporal variability is an important characteristic of wind and solar energies. The EMF27 models represent the implications of intermittent RE sources in a variety of stylized ways, which can have potentially crucial effects on the results. Further research is necessary to develop improved, yet tractable methodologies.

\section{References}

Arvizu D et al. (2011) Direct Solar Energy. In: IPCC Special Report on Renewable Energy Sources and Climate Change Mitigation [O. Edenhofer et al. (eds)]. Cambridge University Press, Cambridge.

Chum H et al. (2011) Bioenergy. In: IPCC Special Report on Renewable Energy Sources and Climate Change Mitigation [O. Edenhofer et al. (eds)]. Cambridge University Press, Cambridge.

Edenhofer O et al. (2010) The Economics of Low Stabilization: Model Comparison of Mitigation Strategies and Costs. The Energy Journal 31.

Goldstein B et al. (2011) Geothermal Energy. In: IPCC Special Report on Renewable Energy Sources and Climate Change Mitigation [O. Edenhofer et al. (eds)]. Cambridge University Press, Cambridge.

IEA (2012) Energy Balances of non-OECD Countries - 2012 edition. International Energy Agency, Paris.

IPCC (2011) Special Report Renewable Energy Sources and Climate Change Mitigation [O. Edenhofer et al. (eds)]. Intergovernmental Panel on Climate Change.

Krey V, Clarke L (2011) Role of renewable energy in climate mitigation: a synthesis of recent scenarios. Climate Policy DOI: 10.1080/14693062.2011.579308.

Krey V, Luderer G, Clarke L (2012) Getting from here to there - energy technology transformation pathways in the EMF27 scenarios. Climate Change, this issue.

Kriegler E, Weyant JP, Et al. (2012) Overview of the EMF 27 Study on Energy System Transition Pathways Under Alternative Climate Policy Regimes. Climate Change, this issue.

Kumar A et al. (2011) Hydropower. In: IPCC Special Report on Renewable Energy Sources and Climate Change Mitigation [O. Edenhofer et al. (eds)]. Cambridge University Press, Cambridge.

Luderer G, Bosetti V, Jakob M, Leimbach M, Steckel J, Waisman H, Edenhofer O (2012) The economics of decarbonizing the energy system - results and insights from the RECIPE model intercomparison. Climatic Change 114:9-37. doi:10.1007/s10584-011-0105-x. 
Pugh G, Clarke L, Marlay R, Kyle P, Wise M, McJeon H, Chan G (2011) Energy R\&D portfolio analysis based on climate change mitigation. Energy Economics 33:634-643. doi:10.1016/j.eneco.2010.11.007.

Rogner H-H, Aguilera RF, Bertani R, Bhattacharya SC, Dusseault MB, Gagnon L, Haberl H, Hoogwijk M, Johnson A, Rogner ML, Wagner H, Yakushev V (2012) Chapter 7 - Energy Resources and Potentials. In: Global Energy Assessment - Toward a Sustainable Future. Cambridge University Press, Cambridge, pp 761-900.

Rose S, Kriegler E, Popp (2012) A Bioenergy in energy transformation and climate management. Climate Change, this issue.

Sathaye J et al. (2011) Renewable Energy in the Context of Sustainable Development. In: IPCC Special Report on Renewable Energy Sources and Climate change Mitigation [O. Edenhofer et al. (eds)]. Cambridge University Press, Cambridge.

Turkenburg WC et al. (2012) Chapter 11 - Renewable Energy. In: Global Energy Assessment - Toward a Sustainable Future. Cambridge University Press, Cambridge, pp 761-900.

Wiser R et al. (2011) Wind Energy. In: IPCC Special Report on Renewable Energy Sources and Climate Change Mitigation [O. Edenhofer et al. (eds)]. Cambridge University Press, Cambridge. 